\title{
Non-invasive foetal heartbeat rate extraction from an underdetermined single signal
}

\author{
Ranjan Acharyya ${ }^{1}$, Neil L Scott ${ }^{1}$, Paul D Teal ${ }^{2}$ \\ ${ }^{1}$ Industrial Research Ltd, Wellington, New Zealand; r.acharyya@irl.cri.nz, n.scottt@irl.cri.nz \\ ${ }^{2}$ Victoria University of Wellington, Wellington, New Zealand; mailto:paul.teal@,vuw.ac.nz
}

Received 17 June 2009; revised 20 July 2009; accepted 23 July 2009.

\begin{abstract}
Extraction of foetal heartbeat rate from a single passive sound sensor on the mother's abdomen is demonstrated. The extraction is based on the assumption that a disjoint band of frequencies exist and foetal signal is concentrated in this band, and further that it can be represented conveniently as a set of wavelet coefficients. The algorithm has been applied to each stream of data obtained from six different channels and the detection performance is elaborated. The algorithm has also been tested on signals from non-pregnant abdomens to show successful rejection of adult heartbeat. The extraction of the desired signal is done in two stages so as to eliminate components from the maternal heartbeat.
\end{abstract}

Keywords: Underdetermined System; Foetal Heartbeat Rate; Wavelet, Blind Source Separation; Non-Invasive; Passive.

\section{INTRODUCTION}

Monitoring of foetal heartrate using a non-invasive technique is still a challenging problem with a variety of approaches $[1,2]$. Usually, foetal heartbeat is monitored using a Doppler ultrasound device which transmits ultrasonic sound waves into the uterus. Most researchers consider that the power used in these devices is perfectly safe, although there are some exceptions [6]. In many cases women prefer that ultrasound is not used on them. Detailed motivation for development and use of accurate, non-invasive techniques for monitoring the foetal heart is covered in the excellent introduction of [1]. This paper describes a method for detection of the foetal heart with a passive acoustic monitoring device (PAM). The process involves collection of signals with microphones and subsequent signal processing.

The passive monitoring of foetal heartrate may be considered as one class of Blind source separation (BSS) problem, which has been an active research area for several decades now. It refers to the problem of estimating the original sources from a mixture. In most cases the mixing system and the number of sources are unknown. Sensors placed on the maternal abdomen provide signals which are mixtures of an unknown number of sources and the nature of the mixing is also unknown. In this section a quick review of other techniques and overview of the technique of this paper is given.

Independent Component Analysis (ICA) is a technique to obtain statistically independent components from a mixture and a solution technique for BSS problems. One way to categorise BSS problems is based on the number of sensor and source signals. Three different scenarios are, the number of sensors are greater than, equal to or less than the number of sources. The third case, which is equivalent to solving for an underdetermined system or for an overcomplete basis, resembles the problem at hand. Detail and an in-depth analysis of algorithms can be found in [3,4,5]. A well known algorithm for sparse sources is known as DUET (degenerate unmixing estimation techniques) [5], which does timefrequency masking. The applicability of the algorithm depends on a W disjoint orthogonal [5] condition of the sources and availability of at least two sensor signals.

Extraction of foetal heartrate using ICA [7] has been attempted in [8] and some interesting results were obtained. In this paper a new algorithm is developed based on certain assumptions on the foetal heartbeat signal. This paper is an extension of an earlier work [9] demonstrating estimation of the foetal heartrate by frequency masking. A crucial difference of this paper compared to [9] is that it avoids filtering of the signal in the time domain. Filtering in the time domain was done in [9] to suppress the maternal heartbeat. The issue of suppressing the maternal signal in the overlap region is addressed in this paper by performing the filtering in two steps. In addition to filtering in the frequency domain the resolution of the signals is enhanced by using a wavelet transformation. The process is discussed in detail in later sections. The method described in this paper requires only 
one mixture provided the sources satisfy certain conditions.

The work reported in $[10,11]$ using SCICA (single-channel independent component analysis) is perhaps the closest to this work in its assumption of disjoint frequency bands for the noises in the mixtures.

In Section 2, the experimental setup and the sensor structure is shown. The assumptions on the sources are explained in Section 3. In Section 4 the algorithm is discussed in detail. Results of the application of the algorithm to a mixture of signals from a pregnant maternal and a non-pregnant abdomen is elaborated in Section 5. Finally, in Section 6 the conclusion and future work are discussed.

\section{EXPERIMENTAL SETUP}

The sensor is similar to that described in [9] which was designed for work using multiple sensors. The sensor structure array is an array of 16 piezo film (PVDF, Polyvinylidene Fluoride) contact microphones of type CM$01 \mathrm{~B}$ in a mat placed against the mother's abdomen. Use of the existing 16-sensor array allowed recording multiple channels simultaneously, although the algorithm proposed here uses only a single sensor signal. The signals are conditioned in a 16 channel low noise amplifier with band pass filtering $(7.2-130 \mathrm{~Hz})$ and then recorded in a data logger. Figure 1 shows the block diagram of the Passive Acoustic Monitor (PAM) and Figure 2 the complete sensor array.

\section{ASSUMPTIONS ON SOURCES}

The model is that the sensor signal is a mixture of signals from multiple sources including foetal heartbeat, maternal heartbeat, other noises internal to the mother's abdomen and external noises from the environment. In addition the mixing system seems to vary with time. The most important assumption is that sound from the foetal heart is band limited and part of the band is isolated from the other sources, including maternal heartbeat. In other words, sources do not share the whole band of the foetal heart beat sound. This conclusion is motivated by the assumption that the characteristics of sound have direct relation to the size of the object generating the sound. The foetal heart is considerably different in size from the maternal heart or any other sound sources inside the maternal abdomen. However, it is expected that there may be overlap of the frequency band of the foetal heart and the maternal heart beat. The maternal heart beat is considered to be the strongest noise present with respect to the desired signal. In addition to being band limited the foetal heartbeat sound is also expected to be compact in the time domain. This implies the signal is assumed to be absent for short periods between heartbeats. Hence, application of wavelet transformation to

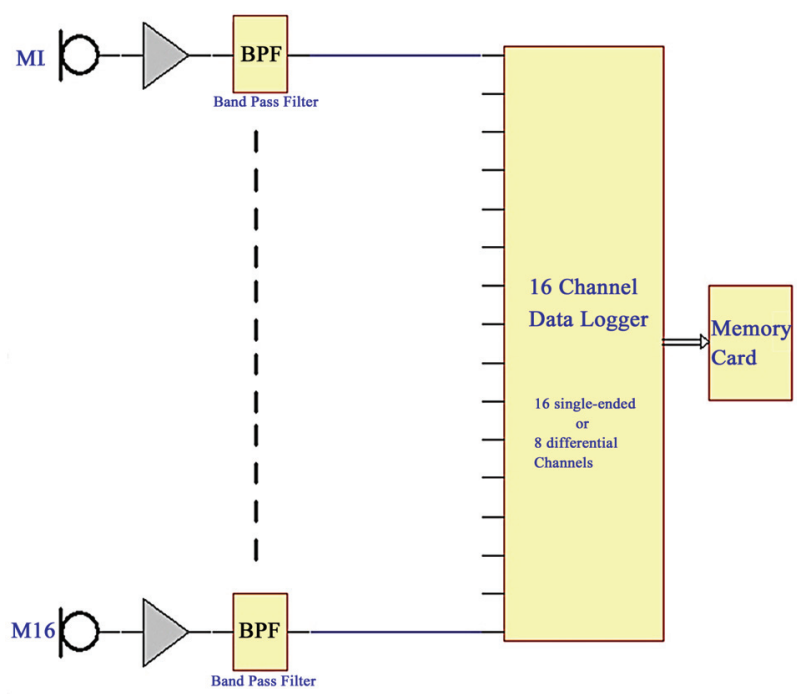

Figure 1. Block diagram of Passive Acoustic Monitor (PAM).

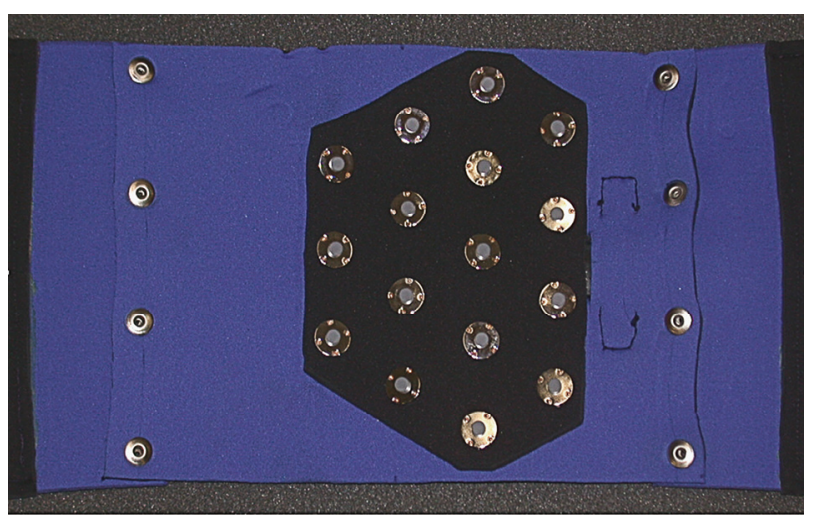

Figure 2. The 16 channel sensor array.

the signal is expected to enhance detection of the heartbeat. The effect of the wavelet transformation will be shown in the next section.

There are further assumptions on the energy content of the signal. In each data block, after initial processing the energy of the processed data in the foetal band needs to be more than a specified threshold value. This is essential to reduce the risk of accepting the adult heartbeat as the foetal one. The energy of the foetal heartbeat needs to be more than a specified threshold value at two different stages. This has a disadvantage that if the signal strength of the foetal heartbeat is very weak then the algorithm can fail to detect it at any particular time. It has been observed that the foetal heartbeat sound is location dependent and it is strong at a certain portion of the maternal abdomen; the strength of the signal is not equal at every point on the abdomen. Further the position where the signal is strongest may vary from time to time.

\section{DETAILS OF THE ALGORITHM}

The algorithm has been developed to exploit the beha- 
viour of the signals given the assumptions mentioned above. The mixture signal obtained from an acoustic sensor is subjected to filtering in both the frequency and wavelet domains. The procedure is an extension of the process discussed in [9], where both maternal and foetal heartbeat were extracted. The algorithm developed here can be extended to extract the maternal heartbeat as well although it is not required for estimation of the foetal heart rate. First, we describe the components of the algorithm and then provide a step by step algorithm description.

The first filtering step is performed in the frequency domain. The spectrum of the mixture signal is multiplied by a profile derived from a Gaussian mixture before inverse Fourier transform. The procedure to derive the profile of the mixture of the Gaussians is as in [9]. The Gaussian mixture so obtained is then modified such that

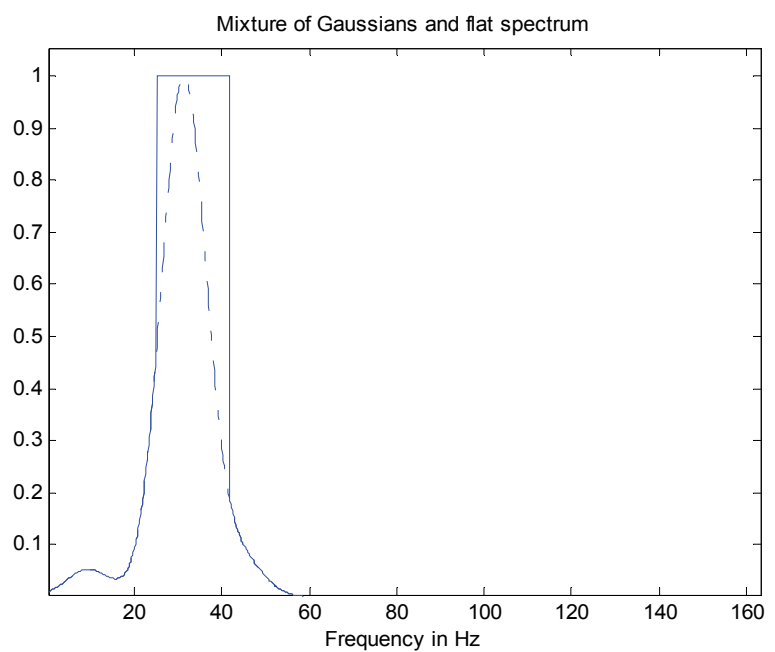

Figure 3. The solid line is mixture of Gaussian and the dashed line is the derived one.

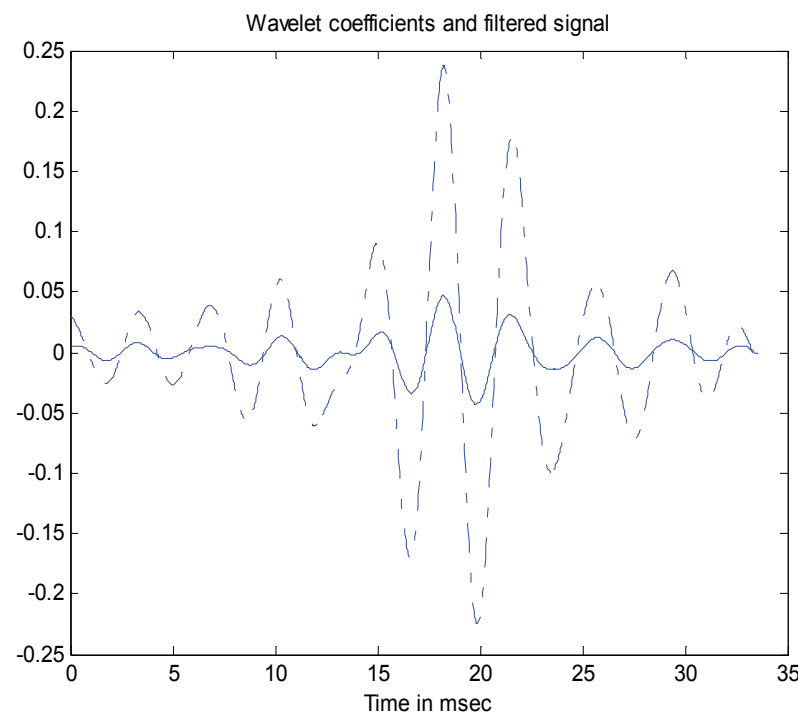

Figure 4. Comparison between filtered signal and its wavelet coefficients (dashed). a range of values around the peak of the mixture are set to unity. This makes the frequency profile flat around the middle portion. The reason for this modification is that the peak energy frequency bin may vary from foetal heart to heart or it may fluctuate from beat to beat.

Therefore a general form of frequency profile in the first stage of filtering seems to be more appropriate. Figure 3 shows the frequency profile derived in [9] and the profile that is utilized in this paper. The mixture signal is filtered by this derived spectrum.

After filtering, the energy of the signal is compared to the energy of the signal prior to filtering. The filtered signal must meet the energy criterion which is set by a specified threshold value, in order to proceed to the next stage of processing, which is carried out for the portions of signals which are expected to have a foetal heartbeat.

Next, a stationary wavelet transform (SWT) of type Discrete Meyer as defined in MATLAB, is applied to the signal. The number of coefficients in each level of stationary wavelet transform has the same number of samples as the original signals. The approximate coefficients of the 5th level are considered [12]. Figure 4 shows a small block of the filtered signal and the corresponding 5 th level wavelet coefficients.

As can be seen in the figure, the portion where the foetal heart beat is expected i.e. higher energy portion of the signal is inflated. Next, the locations of the foetal heartbeat in a block are estimated by determining the peaks of the energy of the wavelet coefficients. The algorithm should detect the peaks corresponding to each heartbeat. Often the change of sign of the derivative is used for detecting a peak. However, the foetal heart signal is essentially oscillatory in nature, each heartbeat event includes several oscillation peaks hence, the change of sign of the derivative would return many peaks which are not of interest. To get rid of this problem the change of sign of derivative algorithm is applied twice. An algorithm returns the first peak estimator as the points of change of sign of the derivative. The same algorithm is then applied to the absolute values of the set of first peaks to determine the overall peak estimates. Figure 5 shows the peaks detected by the algorithm in the first and second stages. The arrows mark the final peaks detected by the algorithm. The two stage peak detector is an alternative to the other peak estimation methods that require a strict threshold value.

The peaks obtained from the second stage are close to the actual maxima, as observed by eye.

The signal around the peaks so selected is expected to be made up of foetal heartbeat signal. At this stage a few peaks may remain from maternal heartbeats. Next, the signal is multiplied by a window function around the peak values. The window function is shown in Figure 6. The purpose of this window function is to attenuate all other sources near the desired foetal heartbeat. Emphasis may be given to the point that the objective of this paper is to recover the foetal heartbeat rate rather than the 


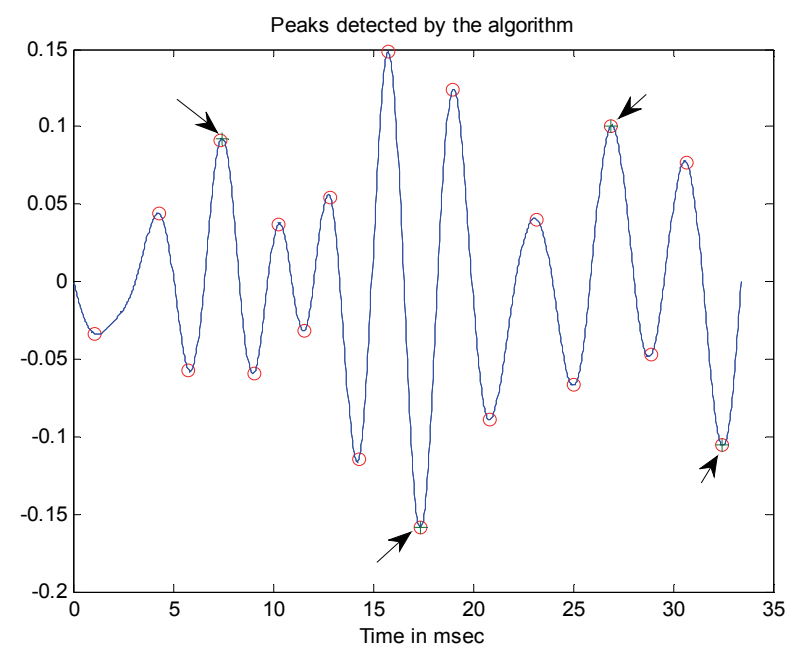

Figure 5. The 'o' are the peaks obtained first and arrow shows the accepted peaks.

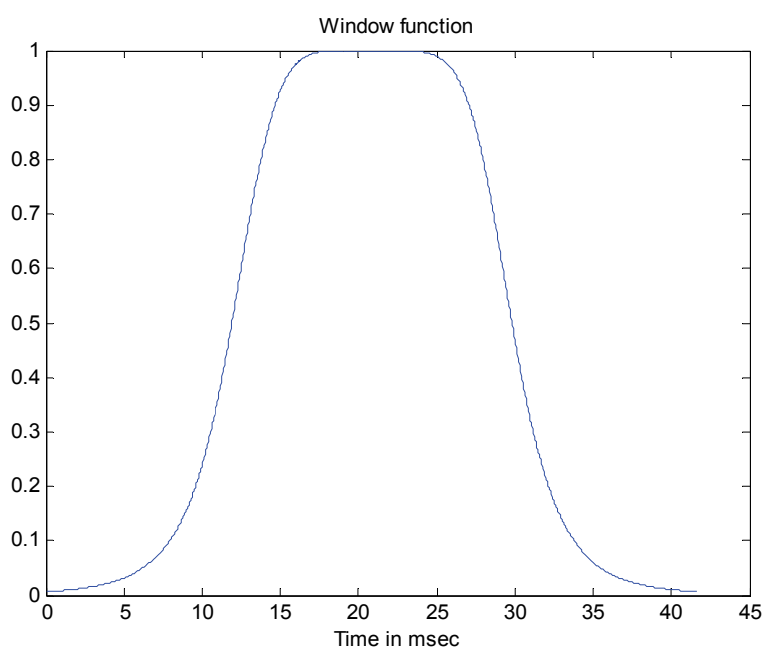

Figure 6. Window to multiply the time domain signal.

waveform. The use of the window function may change the actual waveform of the foetal heartbeat but it is expected not to affect the foetal heartbeat rate. This ends the first stage of the algorithm.

The signal obtained after applying the window function is subjected to another filtering step in the frequency domain. The spectrum of the signal is once again multiplied by a profile derived from a set of Gaussians before inverse Fourier transformation. These new set of Gaussians are obtained in the same way as before [9]. However, the band of the spectrum is shifted towards higher frequencies. For example if the frequency range of the mixture of Gaussians were 15 to 45 Hertz earlier the derived frequency range would be from 30 to 60 Hertz. The motivation for this shifting is to pass the signals which have only foetal heart beat. As mentioned before, the lower part of foetal spectrum overlaps with the maternal heartbeat spectrum. The second step is expected to minimize the energy in the overlap and hence the con- tribution from the maternal heartbeat. To remove the maternal part this second step of filtering is performed. The maternal heartbeat is much stronger than the foetal one and hence this type filtering helped recover the foetal signal from the noise floor. Similar to the previous step, the filtered signal must meet an energy criterion which is set by a specified threshold value, in order to consider it to be a foetal heartbeat. In the final steps the signal is passed through a peak detector twice (like the previous step) so that only those parts of the signal with relatively large peaks can be considered as foetal heartbeat.

The complete steps of the algorithm are given below:

- The data is divided into equal sized blocks. Each of which is filtered by the following steps. In our case the block size is 14400 samples; 6 seconds of data at the sampling frequency 2400 samples per second.

- Multiply in the frequency domain the block of data by the mixture of Gaussians derived as in [9] with flat all pass section at mid band.

- Check the energy content of small blocks of contiguous 2000 samples and compare the ratio of total energy before and after filtering. If a block has a ratio of the energy, after and before filtering greater than the threshold value of 0.005 the block is accepted for the next step of processing. A small block of 2000 samples is chosen instead of 14400 samples so the a whole block of $6 \mathrm{sec}$ data is not lost.

- Perform a stationary wavelet transform and retain the approximate coefficients (low pass filtered part) of level 5 for further processing. The Discrete Meyer wavelet is utilized.

- Find the peaks of each block by passing data through a peak detector twice. The peak detector is based on the change of sign of the derivative of the signal. The peak detector output is passed on if it exceeds the specified threshold value of 0.0067 .

- Multiply the frequency content of the block of data with the spectrum derived from the mixture of Gaussians with flat all pass in the mid band.

- Window the signal with a 1000 sample window about each peak.

- Multiply the frequency content of the block of wavelet coefficients with the mixture of Gaussians and perform an inverse Fourier transform.

- Check the energy content of small blocks of wavelet coefficients of 2000 samples and compare the ratio of total energy before and after filtering. If a block has a ratio of the energy of the signal after and before filtering is greater than the threshold value of 0.1 the block is accepted for the next step of processing.

- Find the peaks of each block by passing data through a peak detector twice. Pass all peaks exceeding a specified threshold value of 0.02 .

- Count the number of peaks per minute to find the heart beat rate. 
- Multiply the signal with a window of 1000 samples so that only selected portions of the energy are kept to produce an audible confidence signal.

\section{RESULTS}

The algorithm was applied to four different datasets. Two sets of data came from pregnant women and two from non-pregnant abdomens. The purpose was to verify that the algorithm can detect foetal heartbeat while not falsely detecting a heartbeat when none is present. Data sets were collected with equipment comprising either 6 or 16 sensor. The sensor elements are identical while the 6 -sensors equipment samples at 2400 data samples per second and the 16-sensor at 300 samples per second. Data sets were regularized by decimating the 2400 data to 300 . As the Gaussian mixture algorithm was designed for 2400 , both data types were then up-sampled to 2400 samples per second. It has been observed that the foetal

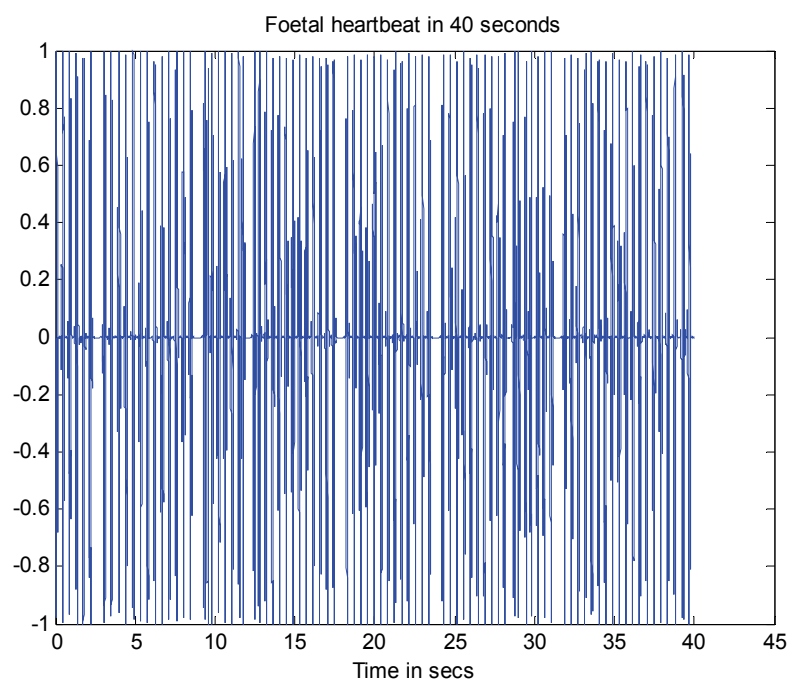

Figure 7. Foetal heartbeat for 40 seconds.

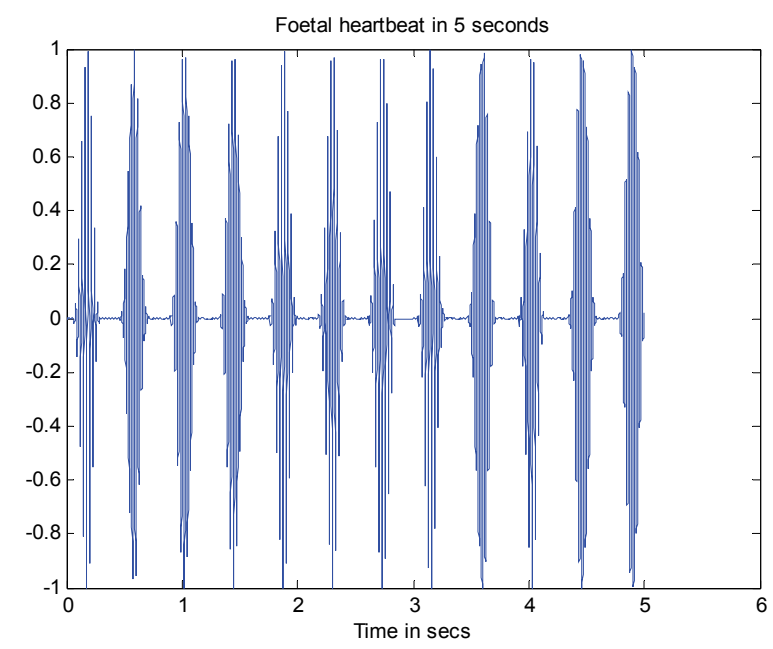

Figure 8. Foetal heartbeat for 5 seconds. heart beat sound is prominent at one sensor at a time. This has been noticed in two different sets of data.

Figures 7 and $\mathbf{8}$ show the foetal heartbeat for a period of 40 seconds and 5 seconds respectively. In the $40 \mathrm{sec}$ ond data one can observe that the foetal heartbeat sound has been missed a few times. From the signals illustrated, a heartbeat rate of 144 beats per minute can easily be estimated. A separate Doppler sensor used at the same time reported a rate between 135 and 160 beats per minute. Similar results were obtained from the other foetal data set.

Data were collected from non-pregnant abdomens for a total of 10 minutes being 5 minutes each from two people. There were 16 channels and altogether the total number of adult heartbeats expected was 11520 assuming 72 heartbeat per minute. The number of false detections as foetal heart beat signal was 154 which is $1.3 \%$ of 11520. In addition the 154 falsely detected pulses were randomly scattered which indicated that they won't contribute significantly to the calculations of foetal heart rate.

\section{CONCLUSIONS}

It has been shown that foetal heartbeat rate can be extracted from a single microphone sensor non- invasively. The rejection of the adult heart rate by the algorithm is established. Future research will look into adjustment of the processing parameters adaptively. In addition the frequency profile of foetal heart beat may be adjusted adaptively.

\section{REFERENCES}

[1] Varady,, P., Wildt, L., Benyó, Z., and Hein, A. (2003) An advanced method in fetal phonocardiography, Computer Methods and Programs in Biomedicine, 71, 283-296.

[2] Kovacs, F., Torok, M., Habermajer, I. (2000) A rulebased phonocar-diographic method for long-term fetal heart rate monitoring. IEEE Trans. on Biomed. Eng., 47(1).

[3] Haykin, S. (2000) Unsupervised adaptive filtering, Vol 1: Blind source separation, Wiley.

[4] Makino, S., Lee, T., and Sawada, H. (2007) Blind speech separation, Springer.

[5] Yilmaz,O. and Rickard,S. (2004) Blind separation of speech mixtures via time frequency masking," IEEE Transaction on Signal Processing, 52(7), 1830-1847.

[6] Kieler, H., Cnattingius,S., Haglund, B., Palmgren, J., and Axelsson, O. (2002) Ultrasound and adverse effects, $U l$ trasound in Obstetrics and Gynaecology, 20(1), 102-103.

[7] Hyvarinen, A., Karhunen, J. and Oja, E. (2001) Independent component analysis, John Wiley \& Sons.

[8] Acharyya, R., Scott, N.L., Teal, P., Deuss, E., and Flierl, J. (2008) Signal separation for non-invasive monitoring of foetal heartbeat, BioMedical Engineering and Informatics. 
[9] Acharyya, R. and Flierl, J. (2008) Blind source separation by time frequency masking of an underdetermined system, International Conference on Bioinformatics and Biomedical Engineering.

[10] Jimenez-Gonzalez, A. and James, C.J. (2008) Blind source separation to extract foetal heart sounds from noisy abdominal phonograms: A single channel method, 4th IET International Conference on Advances in Medi- cal, Signal and Information Processing, MEDSIP'08.

[11] Jiménez-González, A., James, C.J. (2009) Extracting sources from noisy abdominal phonograms: A single-channel blind source separation method, Medical and Biological Engineering and Computing, 47(6), 655-64.

[12] Strang G. and Nguyen, T. (1997) Wavelet and filter banks, Wellesley-Cambridge Press. 